Images du travail, travail des images

6-7| 2019

Femmes au travail\&nbsp: quelles archives visuelles ?

\title{
Filmer le travail à Peugeot Sochaux. Une approche comparative
}

Film work at Peugeot Sochaux. A comparative approach

Salvatore Maugeri

\section{OpenEdition}

Journals

Édition électronique

URL : http://journals.openedition.org/itti/653

DOI : $10.4000 /$ itti.653

Éditeur

Université de Poitiers

Référence électronique

Salvatore Maugeri, «Filmer le travail à Peugeot Sochaux. Une approche comparative », Images du

travail, travail des images [En ligne], 6-7 | 2019, mis en ligne le 01 février 2019, consulté le 14 avril 2021 URL : http://journals.openedition.org/itti/653 ; DOI : https://doi.org/10.4000/itti.653

Ce document a été généré automatiquement le 14 avril 2021

Images du travail, travail des images 


\title{
Filmer le travail à Peugeot Sochaux. Une approche comparative
}

\author{
Film work at Peugeot Sochaux. A comparative approach
}

\author{
Salvatore Maugeri
}

1 Les films sur l'industrie automobile et le travail à la chaîne sont légion. Ils sont œuvres d'information et/ou de propagande entrepreneuriale ou réalisés par des auteurs indépendants, militants, engagés (cf. Hatzfeld et alii, 2007a et 2007b). Le regard posé sur le travail variera évidemment en fonction de ces conditions initiales. Il en va de même des techniques cinématographiques mobilisées (Hatzfeld et alii, 2004). Parmi ces œuvres, deux documentaires tournés il y a respectivement 60 et 42 ans dans les établissements industriels de Peugeot-Sochaux sont accessibles en libre accès sur l'Internet. Ces deux films sont ancrés dans leur époque, celle de la reconstruction, d'abord, où il fallait exalter l'effort productif ; celle de la maturité des Trente glorieuses et de la contestation sociale ouverte par mai 1968, ensuite. La comparaison de ces deux films nous a semblé de nature à révéler certains des artifices mis en œuvre par les cinéastes pour évoquer le travail et conférer au document audio-visuel le pouvoir de conviction conforme aux intentions des réalisateurs et/ou des commanditaires du document. Les enseignements qui peuvent être tirés d'une telle posture comparative ont déjà été éprouvés (Hatzfeld et alii, 2009).

2 Le premier film - En passant par la Franche-Comté ${ }^{1}-, 58^{\prime}$, est le plus ancien. Il a été réalisé en $1954^{2}$, par Michel Boyer, un cinéaste qui semble rompu aux techniques du documentaire ${ }^{3}$. Il est en couleur et répond à une commande de la direction des établissements Peugeot. Son intention n'est pas tant de parler du travail que de faire le portrait d'une entreprise familiale qui a su se rendre maître des technologies pour construire son succès dans une industrie dominant l'économie mondiale. C'est donc une manière d'apologétique de l'innovation technique et du génie entrepreneurial qu'offre ce film pourtant gorgé d'un bout à l'autre de travail humain ${ }^{4}$.

3 Le second document - Avec le sang des autres -, 50', en couleur aussi, plus tardif (1974), est l'œuvre d'un réalisateur indépendant, Bruno Muel, désireux de livrer une œuvre d'auteur. C'est un film militant, affranchi des contraintes institutionnelles et 
s'arrangeant des questions de financement. Le travail et ses conséquences sur la vie des travailleurs sont au centre du propos. Le réalisateur veut relater la peine, l'ennui, l'inhumanité du travail ouvrier dans les usines taylorisés, enchainées, la difficulté qu'il y a à vivre une vie ouvrière, à se faire reconnaître certains droits et certaines aspirations dans un monde dominé par la recherche du profit ${ }^{5}$.

Bruno Muel et le(s) groupe(s) Medvedkine

Si on trouve sur le net peu de traces de Michel Boyer, en revanche les données sur Bruno Muel sont plus abondantes - ce qui n'est pas pour surprendre étant donné sa place dans le cinéma militant. Ce dernier arrive en Franche-Comté, en février ou mars 1967, dans le sillage de Chris Marker, à l'occasion de l'occupation de l'usine Rhodiaceta, à Besançon. La « Rhodia » est une usine de production de fibres textiles synthétiques du groupe Rhône-Poulenc. Elle emploie 3000 salariés (14 000 pour le groupe), presque tous grévistes. Marker est invité par un ouvrier-militantanimateur culturel, Paul Cèbe, appelé à jouer un rôle central dans la naissance du groupe Medvedkine de Besançon puis celui de Sochaux. Le réalisateur venait projeter aux grévistes le film d'Alexander Medvedkine, Le bonheur. Sur place, Marker se lie d'amitié avec plusieurs ouvriers, dont Pol Cèbe. Invitant à son tour d'autres cinéastes et techniciens du cinéma " parisiens » (dont Bruno Muel, mais aussi Jean-Luc Godard, Pierre Lhomme, Mario Marret, Antoine Bonfanti, etc.), Marker sort ses outils, filme la grève, initie, avec ses confrères, les ouvriers aux techniques du cinéma. Le premier groupe Medvedkine est né. De ce séjour à Besançon, Marker tire un premier documentaire : À bientôt, j'espère, 45', diffusé à la télévision française en 1968. Assistés des " parisiens ", les ouvriers réalisent en 1969 : Classe de lutte, un documentaire de 37' sur l'apprentissage du militantisme dans l'entreprise Yema de Besançon. D'autres suivront. En mai 1968, Bruno Muel se rend à Peugeot-Sochaux pour filmer la grève. Un second groupe Medvedkine se crée dont sortira, en 1970, Sochaux, 11 juin 1968, un retour sur la grève, l'occupation de l'usine et les affrontements meurtriers avec les forces de l'ordre en juin 1968 ; en 1971, le groupe produira Les trois quart de la vie (20'), document sur les politiques d'embauche et de logements des ouvriers par la direction Peugeot. Les groupes Medvedkine signeront plusieurs autres documentaires (notamment quatre films intitulés Nouvelle société, numérotés de 5 à 8$)^{6}$. Les deux groupes se dissolvent après 1974 et le tournage des images qui donneront vie à Avec le sang des autres, signé par Muel seul (pour des informations complémentaires sur Muel et les Medvedkine, $c f$. Muel, $\left.2000^{7}\right)^{8}$.

4 Sur le plan formel, l'académisme du premier document ne fait pas de doute. Tous les plans sont tournés avec une caméra fixe, usant de toutes les techniques de prise de vue, à l'exception du portage de caméra. Un sentiment de planification millimétrique du script s'impose. Les moyens filmographiques mis en œuvre permettent l'atteinte des objectifs apologétiques que se sont fixés réalisateur et commanditaire, malgré l'effectif réduit de l'équipe technique.

Dans le second document, la plupart des plans sont tournés caméra à l'épaule, dans le respect des postulats et intentions du cinéma direct, ouvert à l'improvisation, soucieux d'immersion dans le milieu qu'il vient filmer'. Les images peuvent être pour cette raison parfois tremblées, voire bousculées, les zooms maladroits, rapides, les cadrages 
hésitants, les mouvements de caméra intempestifs. On est aux antipodes du parti pris technique mais aussi du climat caractérisant l'autre documentaire. La grande différence vient de ce que la parole est donnée aux ouvriers qui deviennent les véritables sujets du film.

6 Nous chercherons dans cette analyse à mettre en évidence les choix d'écriture cinématographique retenus par les réalisateurs. Nous procéderons à partir de l'étude comparée des images introductives puis d'un examen synthétique des documentaires.

\section{En passant par la Franche-Comté}

7 L'entame du premier film obéit à un script précis, destiné à placer le spectateur dans les meilleures dispositions. Dans les premières images, la caméra est située sur un à-pic et dévoile, par un long plan séquence, un large panorama inondé d'un soleil radieux. En contrebas, une large boucle de rivière ceinturant une petite sommité naturelle écoule paresseusement ses flots. On aperçoit un petit village, tandis que des sons de cloches, des chants d'oiseaux émergent soudain du silence. On pourrait être n'importe où dans une campagne française. Deux jeunes gens, un peu scouts - on les verra en shorts et sacs à dos -, apparaissent alors à l'écran et contemplent la splendeur de la nature.

Illustration 1

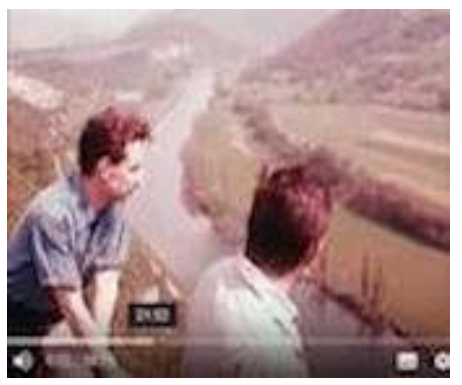

Illustration 2

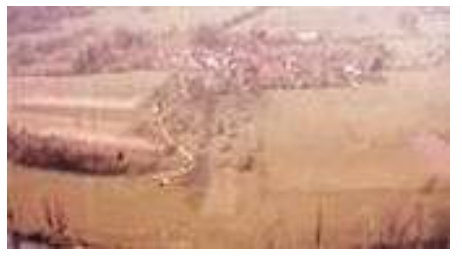

Illustration 3

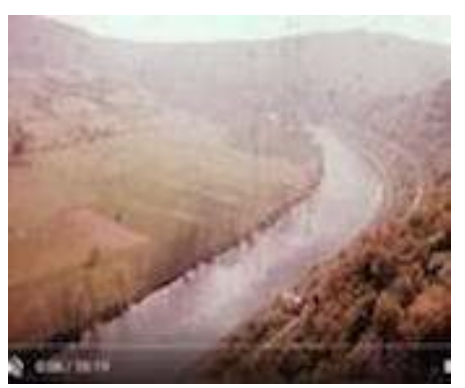


Dans le plan suivant, les jeunes ramassent leur sac-à-dos et se mettent en route. L'un d'eux sort un harmonica de sa poche et une musique entraînante se fait alors entendre. C'est une imitation d'En passant par la Lorraine, marche militaire sonore qui s'harmonise parfaitement avec le soleil illuminant cette belle journée.

9 À la $42^{\mathrm{e}}$ seconde, le générique est lancé, la caméra fixe un plan de route ensoleillée. Du fond de l'écran émergent nos marcheurs. Le nom de Peugeot est le premier à s'inscrire. Suit la liste habituelle de l'équipe de tournage - très courte -, puis le titre.

Illustration 4

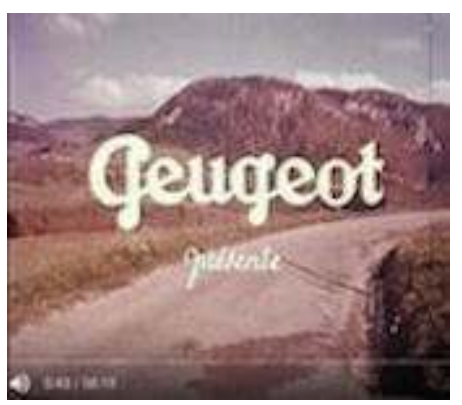

Illustration 5

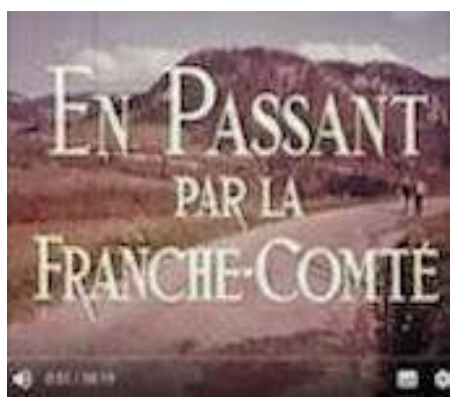

Illustration 6

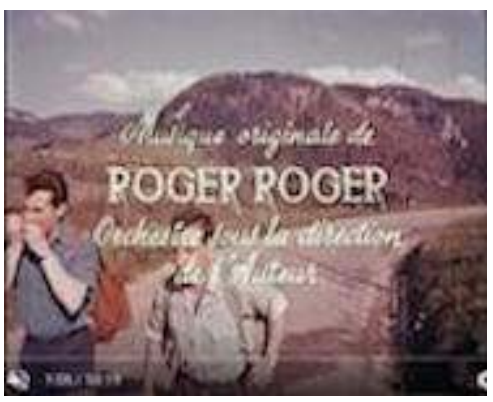

Tous les plans ultérieurs de l'introduction, montrant les deux jeunes musardant le long de petites routes montueuses au son d'une musique dynamique, conforteront l'impression de vigueur et de bonheur imprimée par l'entame.

11 Empruntant un chemin détourné - la traversée fortuite du Doubs par deux jeunes marcheurs en route pour la Suisse -, l'introduction vise à inspirer un sentiment de confort et de détente par le spectacle d'une nature ensoleillée et une ambiance de farniente, de liberté et d'ouverture à ce que le monde a de meilleur. Dans le même temps, il s'agit d'introduire dans l'inconscient du spectateur le thème implicite de la 
route, de la liberté et de son vecteur: l'automobile. On a vu se succéder les plans de départementales sous divers angles, en utilisant la plupart des techniques de la prise de vue, aussi bien des plans fixes larges ou rapprochés, que quelques mouvements discrets de caméra suivant des véhicules en déplacement. Plusieurs vues larges ou en plongée ont donné à la prise de vue une certaine majesté, façon carte postale.

Illustration 7

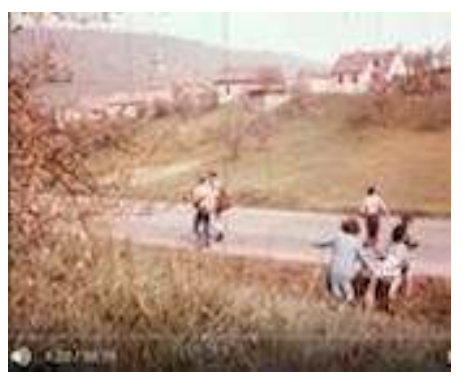

Illustration 8

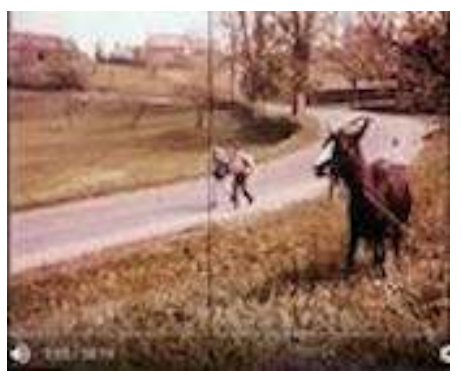

Illustration 9

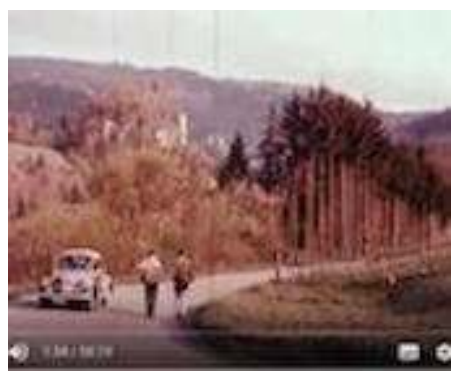

Une sensation de disponibilité, de vacances, au double sens du terme, l'impression que tout est possible sont conférées aux images par la place qu'occupent la nature, les animaux, les plans panoramiques et le refus d'insérer, au départ, la parole humaine dans le récit, privilégiant les bruits ambiants ou des musiques suggestives. Un tel choix permet au spectateur de laisser aller son imagination, construire sa propre flânerie aux côtés de ces deux jeunes marcheurs au cœur de la campagne française, sans s'interroger sur le but du voyage.

13 La rupture de rythme intervient quand un automobiliste, au volant d'une Peugeot 403 rutilante, toit ouvert, propose aux marcheurs de les prendre à bord (1'54"). 
Illustration 10

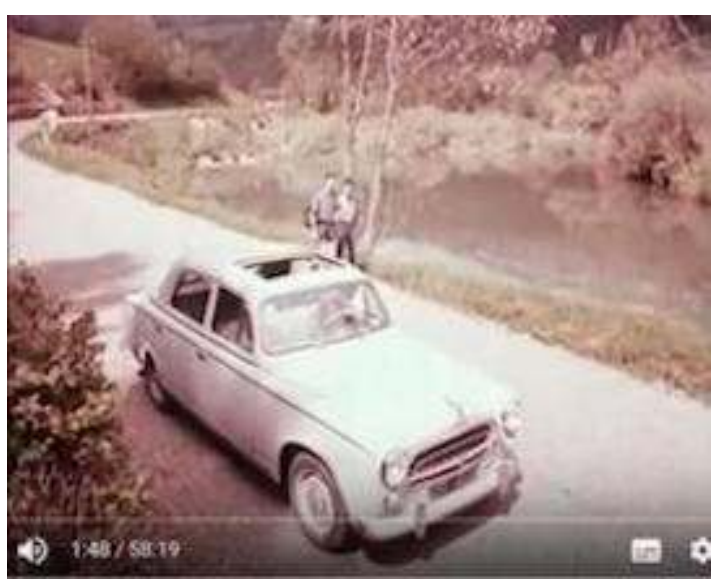

Illustration 11

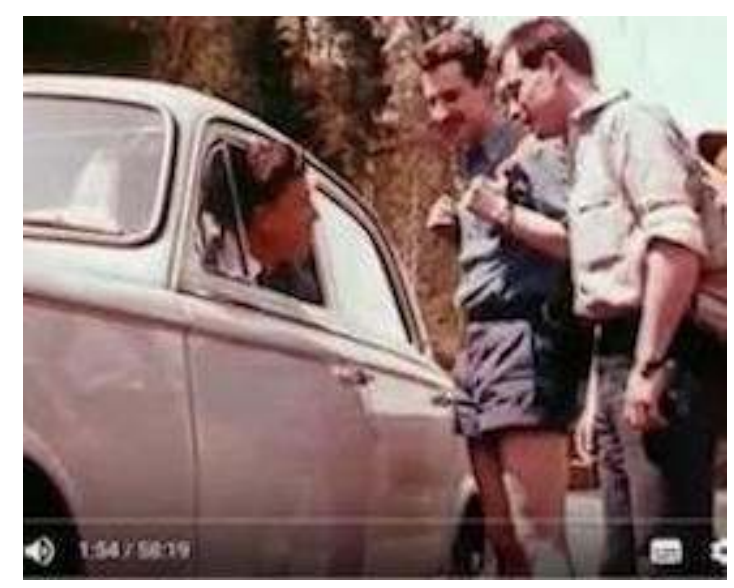

Dès que la voix humaine se fait entendre, le temps s'accélère. La marche cède la place à la locomotion motorisée. La technologie relaie et accélère le corps humain au son d'une musique flûtée, cette fois, rapide toujours, plus intimiste cependant, car on se trouve à bord du véhicule. Filmés en vue subjective au démarrage de la voiture, les plans paraissent plus vifs. La campagne est traversée à vive allure. L'arrivée en ville (2'26"), marquée par une succession de plan rapide (apparition d'un panneau indiquant l'entrée de Montbéliard, vue plongeante sur le château médiéval situé sur la commune, etc.), imprime une cadence précipitée aux événements.

Illustration 12

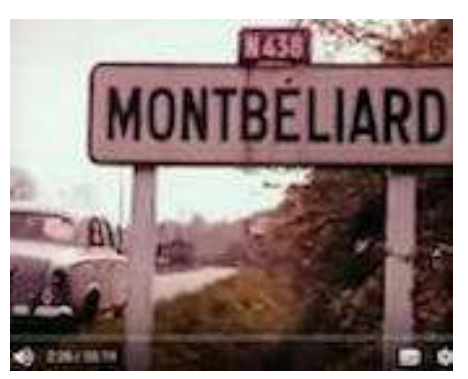


Illustration 13

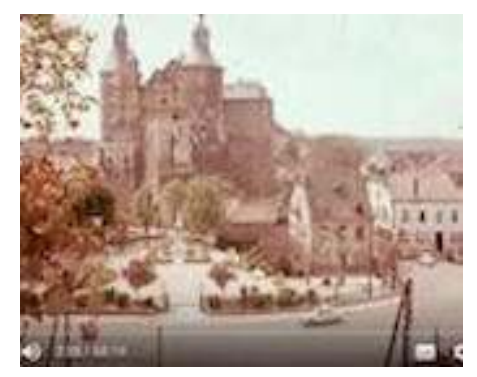

Illustration 14

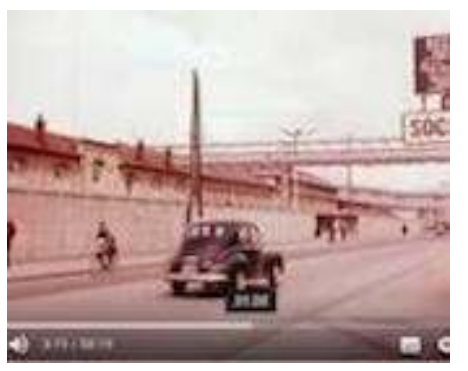

On est passé d'un instant de la campagne semi-déserte à la ville. C'est qu'il faut tout à la fois en venir au sujet du film et préparer le spectateur à la suite du documentaire.

Dans le plan suivant, après quelques hectomètres de route et un nouveau diminuendo musical, la voiture s'arrête après avoir longé un mur d'enceinte derrière lequel on aperçoit les sheds d'une usine. Le pilote annonce qu'il est arrivé au terme de son parcours : «C'est là que je travaille : aux usines Peugeot. » Des bruits de trafic montent aux oreilles, un bout de panneau indicateur laisse apparaître le nom de Sochaux, tronqué. En voix off, un des randonneurs s'extasie : «Les usines Peugeot! Ça doit être intéressant. Peut-on les visiter?».

17 Le sujet du film est enfin lancé. Il s'est écoulé 3'30" avant que nous ne pénétrions dans l'enceinte de l'usine.

\section{Avec le sang des autres}

Les premières vues de l'entame de ce film sont difficiles à décrypter. Quelques secondes avant la première image, on entend une musique : un air à trois temps, entraînant, joué par des cuivres. Cette entrée en matière contraste avec ce que nous révèle le premier plan : une esplanade humide, des hommes emmitouflés dans des vêtements d'hiver, parfois coiffés d'une casquette, d'un béret ou abrités sous un parapluie, se protégeant de quelque chose comme du crachin. On ne comprend pas ce que ces gens font là. Le seul indice, c'est en arrière-plan, le mur d'enceinte, la façade et les toits d'une usine. L'image est en couleur, mais tout semble gris, terne, passé. Le sol est noir, parsemé de flaques d'eau. Lentement, la caméra détaille ce décor triste d'hommes disposés en rang d'oignon sur ce parking. Le panoramique donne une idée de la longueur de l'usine en arrière-plan. 
19 Au terme du mouvement de caméra, on découvre d'où vient la musique : une fanfare de jeunes musiciens en tenue de circonstance (vestes écarlates, chapeau haut de forme, etc.) et à cheveux longs qui, côte à côte, soufflent dans leurs instruments.

Illustration 15

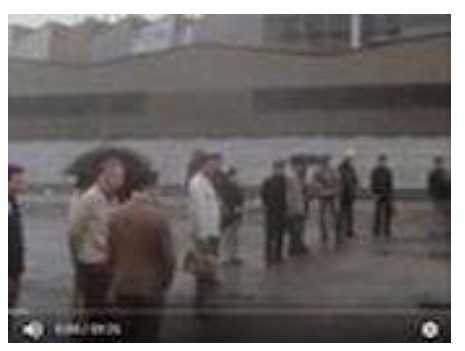

Illustration 16

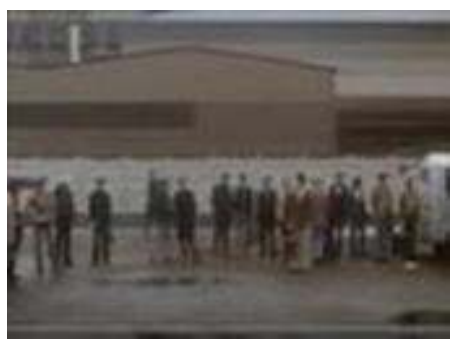

Illustration 17

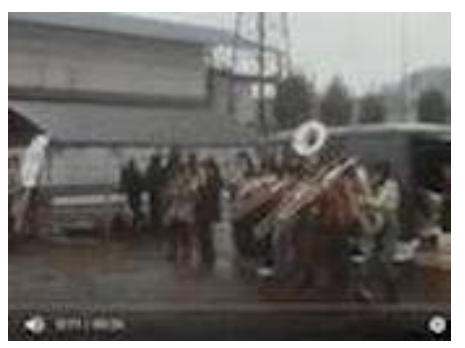

Qu'est-ce que fait cette fanfare sur ce parking humide et glacial ? Où est-on, qu'est-ce qui se prépare? On ne sait pas et on ne saura pas.

21 Ce qu'on découvre ensuite, c'est la fièvre, la presse propre aux sorties d'usines. La musique s'est arrêtée. La caméra, soudain, fait face au portail d'une usine. Un grondement sourd s'entend. On voit un essaim d'ouvriers s'échapper, telles des abeilles, à pied, à vélo, en mobylette, rapidement, résolument. Il y a quelques voitures. Toute cette foule mouvante, ces engins motorisés nous arrivent dessus à travers la brume sale de cette sale journée. Les piétons ont les mains dans les poches de grosses vestes ou de manteaux, tandis qu'on voit des gants aux mains des pilotes des deux roues. Ne s'entend que le bruit des engins qui fuient l'usine. Puis, la musique repart, la même musique que dans le premier plan, mais aux instruments se sont jointes plusieurs voix à l'unisson pour un même chant. Les paroles ne sont pas claires, le bruit des moteurs au sortir de l'usine couvre les voix. On entend les mots : " moulin de la Galette », "vie », « ouvriers ", " paysans ». Une tache blanche apparaît alors au milieu de l'écran, comme issue de la brume et qui grossit, se précise, se comprend: c'est le titre du film qui s'avance vers nous, ainsi que les ouvriers jaillissant de cette géhenne mystérieuse. On 
commence à deviner les lettres: Avec... Avec le sang des autres. Le titre est à présent installé au milieu de l'image. Et tout est dit déjà.

Illustration 18

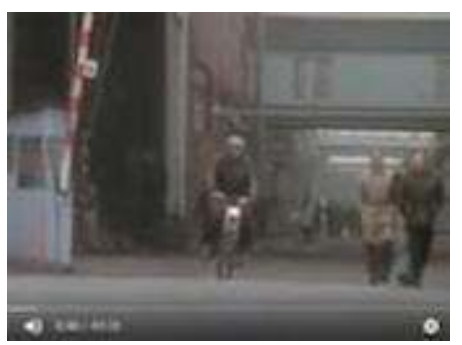

Illustration 19

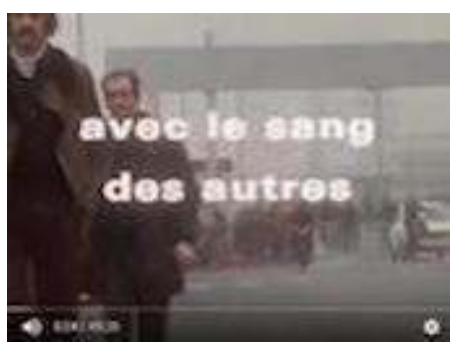

Illustration 20

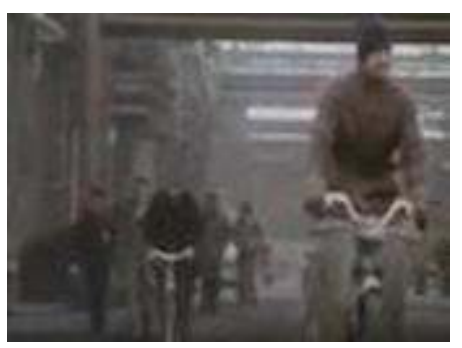

Le démarrage de ce film est totalement opposé au premier. On ne cherche pas à raconter une histoire dans un cadre naturel riant afin d'introduire par la bande le sujet $\mathrm{du}$ film. On est plongé directement dans l'agitation mécanique la plus violente, la plus mordante, à la fois en raison du froid qui agresse les corps et de la frénésie propre aux sorties d'usine, quand les ouvriers se pressent de rejoindre la chaleur du nid. Parallèlement, un élément d'étrangeté est introduit par la présence de la fanfare sur l'esplanade de stationnement des bus. L'entrain propre à la musique, la bigarrure des tenues des musiciens, l'éclat sonore et visuel des cuivres, la chaleur des chœurs contrastent avec la grisaille d'une sortie d'usine hivernale. Quelque chose de la culture contestataire se laisse deviner dans les images, les sons, une pancarte brandie par un ouvrier, l'inscription 1er mai, les paroles de la chanson qu'on devine. Mais que fait cette fanfare, quel événement est-elle venue célébrer? Nous ne le savons pas et rien dans l'attitude des ouvriers qui, avant de rejoindre leur car, s'arrêtent un instant, ne permet de tirer de conclusion. Nous ne saurons pas ce que font ces musiciens ici, quelle manifestation a été organisée à leur intention au moment du changement d'équipe.

La caméra a donné à voir tantôt les ouvriers face à l'orchestre, sur un large espace humide, les visages fermés, le cheveu souvent long, la cigarette au bec, la tête couverte 
de casquettes, de bérets, les cols bien fermés, la caméra détaillant parfois les visages, les regards, le silence de ces hommes écoutant la musique.

Illustration 21

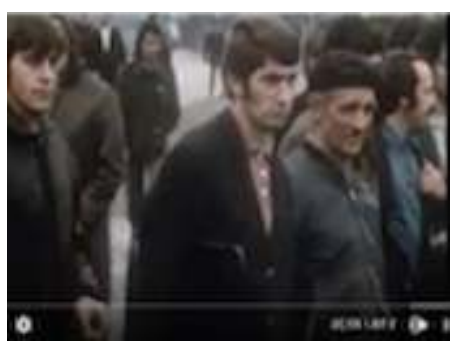

Illustration 22

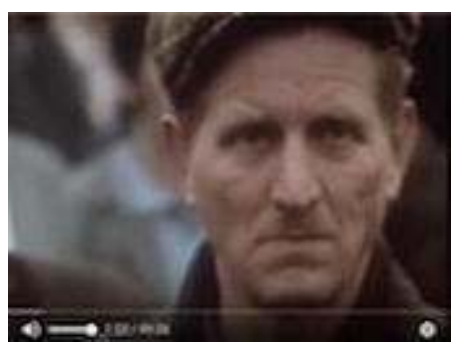

Illustration 23

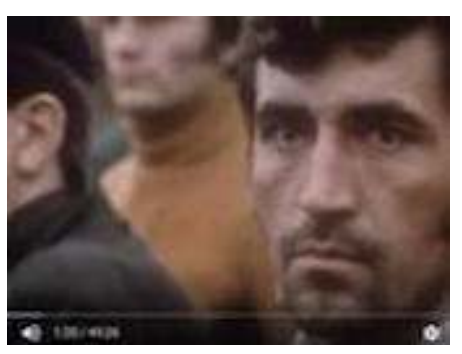

Tantôt, elle a braqué son œil enregistreur sur la sortie de l'usine, laissant voir la dimension génésique de l'usine qui, pareille à la gueule d'un fût de canon, crache ses ouvriers. Tout est réuni pour donner l'impression de chaos, d'agitation, de frénésie. C'est l'hiver et la musique de la fanfare sur ces images crée un contraste inconfortable. Ces deux choses ne vont pas ensemble, cette sortie d'usine fumante et hivernale, cette musique entraînante : ça ne colle pas. Et puis, autre malaise : où sont les femmes? Cette sortie d'usine est masculine, exclusivement. Les femmes sont ailleurs - où ?

2'41" contre 3'30" pour le premier film - pourtant plus courte, l'introduction du second film semble durer plus longtemps, dire plus de choses, quoique moins facilement interprétables. Dans le premier film, la promenade en Franche-Comté est lisse, linéaire, enjouée, le script introductif a été pensé et appliqué sans improvisation. L'objectif était clair : arriver devant l'usine, faire comprendre qu'on allait la visiter en compagnie de deux jeunes gens sympathiques et curieux. Dans le deuxième film, l'intention n'est pas claire. On peut même faire l'hypothèse qu'il n'y en a pas; plutôt que dire quelque chose, on veut donner à voir, à sentir : on s'adresse aux nerfs, l'intellect est laissé de côté. C'est à une expérience sensorielle qu'on est invité, pas à un cours bien ordonné où on nous expliquera comment des machines fabriquent d'autres machines grâce au 
génie des ingénieurs. Ce qui compte ici ce sont les hommes. Les hommes et leurs sales affaires.

\section{Le script et l'écriture cinématographique}

\subsection{En passant par la Franche-Comté}

Qu'on ne s'y trompe pas, En passant par la Franche-Comté est un modèle du genre. Le didactisme dont fait preuve le script peut facilement en faire un exemple.

Après les avoir quittés face à l'usine, on retrouve les jeunes gens dans un bâtiment, devant une bière. Le parti pris de détente auquel nous a habitués l'introduction est confirmé. L'automobiliste présente les jeunes à un salarié, M. Lebrun, qui va leur servir de guide. La direction a donné son accord pour cette visite impromptue. Quoi de plus normal, en effet? M. Lebrun arbore une longue blouse blanche, une cravate, des lunettes. Il entraîne les jeunes, mais d'abord - magie du cinéma - la visite commence par un cadrage géographico-historique, avec voix off, cartes et animations. Localisation et genèse des différents établissements de l'entreprise en Franche-Comté sont précisées une à une et illustrées par des prises de vue aérienne ${ }^{10}$. On rappelle l'ancienneté de l'engagement industriel des Peugeot, arrivés à la sixième génération d'entrepreneurs. Date d'ouverture des sites, superficies occupées, nombre de salariés, tonnages de charbon et nombre de kilowattheures consommés, tous les chiffres de nature à édifier les deux jeunes - et à travers eux, le spectateur - sont précisés dans ce petit préambule qui se conclut par la présentation du site de Sochaux, objet de la visite ${ }^{11}$.

Illustration 24

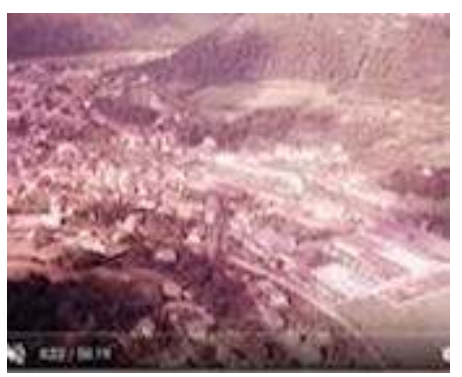

Illustration 25

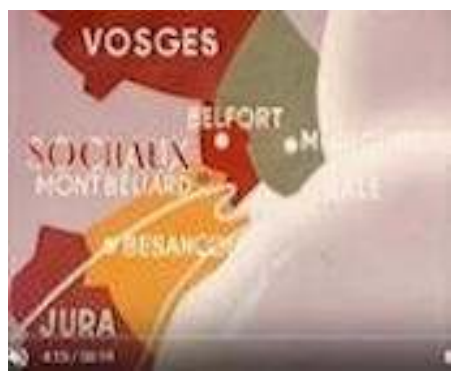




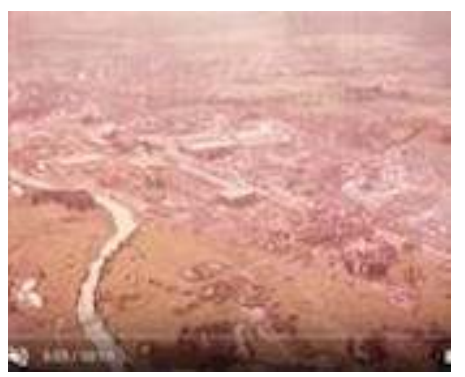

Cette visite s'organisera de façon méthodique en parcourant l'ensemble des ateliers dans l'ordre du flux des marchandises dont l'assemblage accouchera d'une voiture, ici la 403. Le procédé est classique dans les films d'entreprise (Hatzfeld et alii, 2006). Elle commence par la forge, où sont coulées les pièces d'acier du moteur. La camera s'arrête longuement sur les postes de travail les plus significatifs, filmant les organes d'acier qui estampent, ébarbent et plient le métal aux formes voulues tandis qu'une voix off débite les commentaires adéquats. Les deux jeunes gens et leur guide ont disparu du champ, on les retrouvera de temps en temps, au cours de la visite, penchés sur un outil, admirant un procédé, montrant du doigt un organe mécanique, etc. À compter de ce moment, le héros du film c'est l'usine, ce sont les machines, l'intelligence des procédés, la justesse et la précision des mécanismes, la minutie et la rigueur des contrôles. Les chiffres tombent, régulièrement: " 4000 pistons/jour ", " 350 tonnes de tôle/an ", "précision au micron ", marteaux de " $5500 \mathrm{~kg}$ de masse tombante ", etc. Il en ira ainsi jusqu'à la fin du documentaire. Chaque secteur est l'occasion d'une apologie de l'excellence technique de la marque. Parfois, le commentaire laisse la place à une question posée, hors champ, par un des deux jeunes, qui entraîne la réponse enthousiaste de M. Lebrun saluant l'avant-gardisme de l'entreprise.

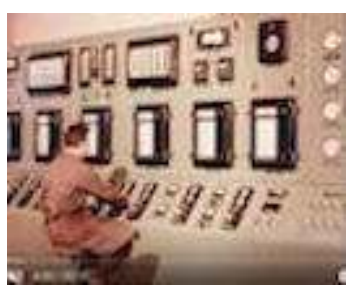

Illustration 28

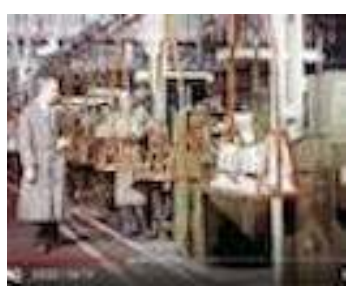


Illustration 29

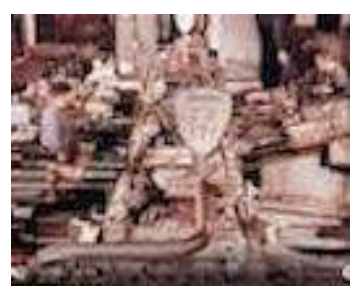

Illustration 30

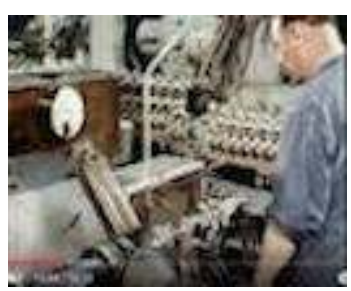

Illustration 31

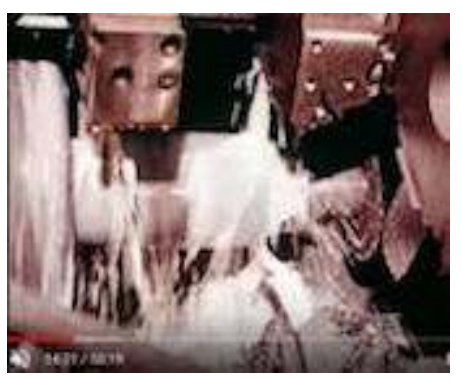

Illustration 32

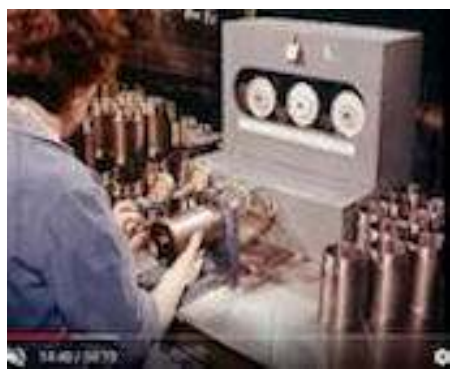

Illustration 33

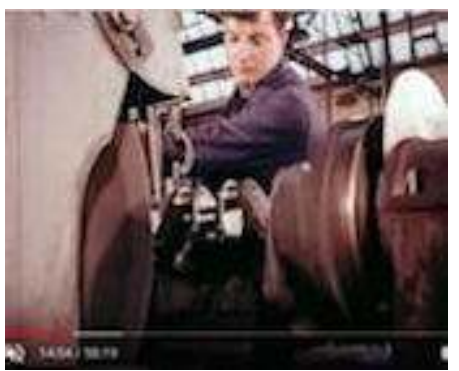

Sur le plan visuel, il faut souligner la beauté, la plasticité des images, la justesse des cadrages et des cadences. La visite se fait au pas, lentement, le spectateur a tout le loisir 
de détailler les outils qui martèlent, forent, liment, abrasent, soudent au milieu des huiles grasses et irisées qui attendrissent les métaux ou des gerbes d'étincelles venant allumer de petits quatorze juillet dans les ateliers. La lumière partout est transparente, l'air clair et désencombré. Partout, aussi, la propreté. Tout semble neuf, astiqué, rutilant. Les machines, le sol aussi et jusqu'aux vêtements des salariés qui sont repassés de frais. Rien ne traîne, rien ne gêne. La sensation d'espace est sensible. Il faut noter aussi ce fait insidieux que la bande son est tamisée. Les ateliers ne sont pas secoués par les presses de 2000 tonnes qui enfoncent les plaques d'acier ou les outils qui arrachent au métal ses copeaux. Les lames, les forets, les dents et les pointes pénètrent la matière avec douceur, dans une ambiance apaisée. La voix off insiste d'ailleurs beaucoup sur les espaces de silence réservés aux contrôles, dans lesquels se glisse la caméra pour quelques secondes de totale quiétude. Une impression de douceur, de calme s'éprouve à la vue du travail, où parfois apparaissent un cadre, un contrôleur, en blouse grise ou bleue, certes, mais en cravate. Très souvent, le travailleur lui-même disparaît. Tout au plus voit-on sa main, rapidement - une main toujours propre - qui charge une machine ; mais le vrai sujet, c'est la machine, la machine et ses « outils» : c'est elle qui garantit la perfection et la tranquillité du client. Il est tout à fait surprenant de constater le peu de cas que fait le commentaire de la présence humaine dans les ateliers. Certains plans pourtant ne peuvent éviter de rendre le grouillement humain qui anime certaines chaînes, mais en dehors de rares, très rares occasions où le savoirfaire ouvrier est salué, les hommes sont oubliés, négligés, invisibilisés - même quand ils sont cinq, dix sur le même plan - au profit de la machine, seul sujet de l'effort démiurgique qui est conté1 ${ }^{12}$.

Plus absent encore que l'homme, la femme. Si les premières minutes de la visite n'offrent à la vue que des mâles au travail, en bleu, couverts d'un tablier, quasiment tous porteurs de bérets et souvent la cigarette au bec, l'apparition de la première silhouette féminine dans un des ateliers de contrôle, à la 14'30" minute, ne suscite aucun commentaire particulier. Pourtant, après cette première femme, beaucoup d'autres se mêleront aux hommes dans la fabrique de la 403, y compris sur certaines chaînes, mais le fait n'est jamais relevé par le commentateur. Rien de surprenant. Pas plus que les hommes, elles ne sont le sujet du film. Le centre d'intérêt, c'est l'usine, ses technologies, ses processus et, en filigrane, ceux qu'on ne voit jamais : les ingénieurs, qui disposent des ressources d'intelligence situées au principe de cette merveilleuse mécanique usinière, capable comme dans une fable de transformer le plomb en or. Car c'est l'histoire d'une création qu'offre ce documentaire, celle d'un enfantement, d'une métamorphose magique où la matière informe livrée par la nature se transforme en objet de luxe et outil de liberté, ainsi que le rappellera la conclusion du film qui voit nos deux jeunes marcheurs de nouveau sur une route resplendissante de soleil, étirant sa ligne de fuite vers les lointains, au son de l'orchestre et de l'harmonica que l'un d'eux a tiré de nouveau de sa poche de chemise.

31 Modèle du genre disions-nous sur le fond comme sur la forme. Il faut dire rapidement la variété des procédés filmiques engagés par l'équipe de tournage. Toutes les techniques de la prise de vue sont mobilisées avec art et à propos, du plan fixe sur un organe mécanique en mouvement, jusqu'au travelling avant ou arrière, sans oublier les plongées et contre-plongées, les panoramiques, les zooms avant et arrière. Toutes ces techniques sont réquisitionnées, jamais gratuitement, toujours avec justesse et parcimonie. Le sentiment de maîtrise, de souveraineté technique que veut rendre le commanditaire à propos de son usine est suggéré avec brio par l'équipe de réalisation 
qui joue de toutes les ressources cinématographiques. S'il faut descendre dans une fosse pour filmer de dessous les véhicules au moment des derniers contrôles, la caméra n'hésite pas, offrant au spectateur les mêmes perspectives que les hommes au travail, mais sans la sensation d'enfermement, d'étouffement qui accompagne certains métiers particulièrement confinés. À l'inverse, s'il est besoin de s'élever pour offrir une vue plongeante sur une machine, une chaîne, etc., la caméra trouve le bon emplacement, en hauteur, et joue admirablement des effets de profondeur pour suggérer espace et plaisir.

Là où le travail devient harassant, l'espace rétréci, la presse, la cadence bien visibles, là où la caméra ne peut faire autrement que de montrer le travail, la peine, alors, on usera d'un subterfuge : la musique. Dans l'atelier dit d'habillage/finition, long de $600 \mathrm{~m}$, où les caisses sorties de la peinture seront dotées de leurs équipements intérieurs avant d'être associées aux organes moteurs, train et transmission, c'en est fini de la lenteur. Il faut s'employer, user de mille mains et contraindre les corps à des positions aberrantes dans des espaces parfois si rétrécis et confinés qu'il a fallu trouver une solution pour alléger la sensation d'inconfort que pouvaient entraîner ces séquences harassantes. Un air de jazz cubain enlevé vient alors égayer les images (43'44"). Au rythme des syncopes et des percussions, les travellings se succèdent le long de la chaîne, passant en revue différentes opérations de montage, habillage, finition, etc. Le commentaire se fait plus espacé.

Illustration 34

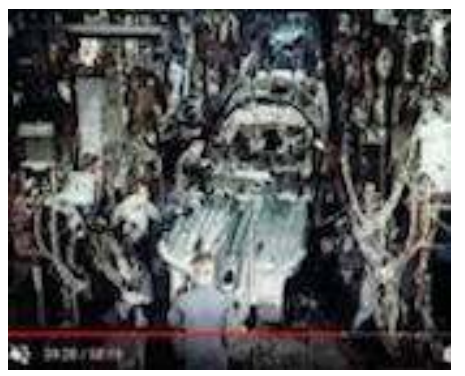

Illustration 35

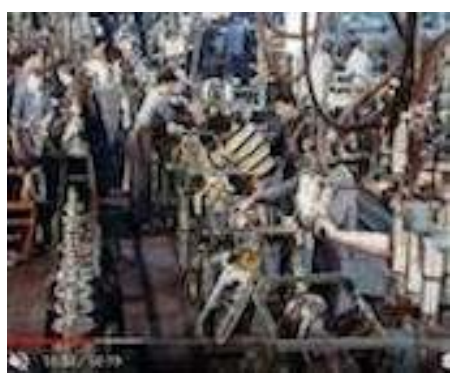


Illustration 36

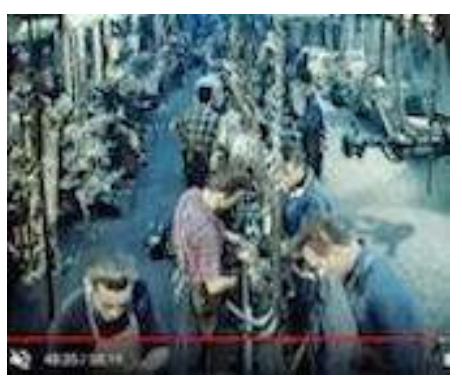

Ce que les images ne peuvent cacher, malgré la musique, ce sont les hommes pliés dans des habitacles trop petits, engagés deux, trois, quatre à la fois, pour fixer du tissu au toit de la voiture, monter un tableau de bord, installer les câblages électriques, dans ce qui semble être une jungle inextricable de fils et de pièces détachées. On aperçoit parfois des corps assis, couchés dans le coffre de la voiture, à l'avant, à l'arrière, afin d'exécuter une opération dans un recoin impossible (44'12" et suivantes). Le jazz est là pour nous entraîner dans cette danse avec la matière, mais le commentaire reste muet sur les acrobaties auxquelles sont contraints les hommes.

\section{Illustration 37}

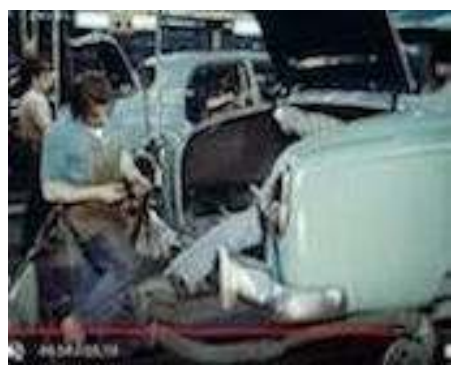

Illustration 38

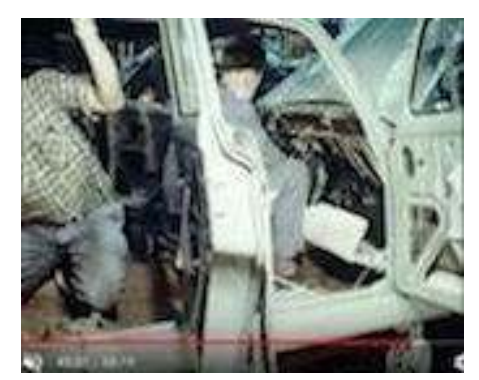

Illustration 39

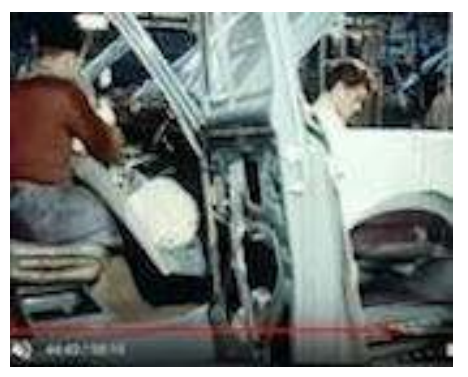


34 À la 48'41", quand la caisse enfin "habillée » rejoint la chaîne où l'attendent son moteur, son train, ses transmissions, le jazz laisse la place à la musique classique. Une entrée tonitruante de cuivres, façon jardin de Versailles, marque l'arrivée d'une caisse suspendue à un palan électrique. Le moment est solennel, quasi-matrimonial. C'est l'accouplement de la caisse et de ses organes de locomotion. Ce qui n'était qu'une virtualité inscrite dans la tête des ingénieurs va enfin s'incarner dans un objet doté de fonctionnalités précises.

Le documentaire est absolument complet. On a assisté, méthodiquement, à la fabrication d'une voiture, des lopins d'acier sortis de la forge jusqu'au véhicule en phase de chargement sur les convois ferroviaires pour la livraison au client. Durant tout le film, les gestes sont rendus avec beaucoup de réalisme : on est au bord de la chaîne, à côté de l'ouvrier et son travail, on le comprend, on le sent, on l'éprouve, mais il n'est jamais salué comme un exploit. Cette implication, ces engagements corporels, cette répétition journalière de gestes stéréotypés semblent aussi naturels que notre fonction respiratoire ou le vol des oiseaux. La condition humaine des ouvriers d'usine, rivés à la sempiternelle répétition des gestes, dans les ateliers taylorisés, le long des chaînes, ne donne lieu à aucune interrogation et même à l'atelier de peinture, où l'on a vu des hommes, pistolet de peinture à la main, dans des espaces saturés de vapeur chimique travailler sans masque, la question des risques professionnels, de la santé et de la sécurité au travail est balayée d'un revers de main par M. Lebrun : pas besoin de masque, pensez-vous, nos cabines sont dotées de ventilateurs puissants. Le travail, c'est la santé. Cela saute aux yeux.

On comprend que nos jeunes gens, au sortir de la piste d'essai où, au son d'un air endiablé, ils ont enchaîné les tours et les mises à l'épreuve d'un véhicule tout juste accouché par la chaîne, repartent le cœur léger sur la route des vacances. Le monde est un jardin d'Eden où, grâce au génie industriel, poussent les automobiles qui nous emmènent au bout du monde.

\subsection{Avec le sang des autres}

Dans ce film, tout est différent. On le comprend dès les premières images qui suivent le générique. Le spectateur est d'emblée plongé dans l'enfer du travail. Un grondement furieux et des éclairs électriques bleus sautent au visage. On découvre un ouvrier revêtu d'un casque de soudeur, penché sur son ouvrage, tel un robot de série B, tandis que fusent les étincelles allumées par son appareil. Sa tête dodeline en cadence, son regard invisible derrière le verre fumé prend régulièrement connaissance de l'état de sa soudure. Il est seul aux prises avec la matière tandis que le bruit de l'usine, les agressions sonores, visuelles, olfactives aussi, accompagnent sa tâche. Le travail sent, le travail aveugle, le travail vrille les tympans et chaque image le donne à voir, à entendre, à comprendre - sans le moindre commentaire, procédé d'emphatisation déjà choisi, en 1972, par Louis Malle dans Humain, trop humain ${ }^{13}$. 
Illustration 40

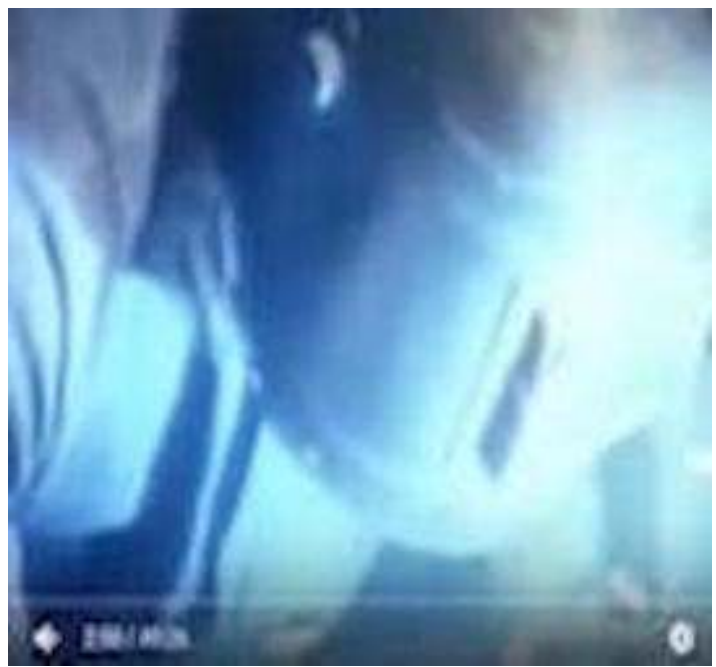

Illustration 41

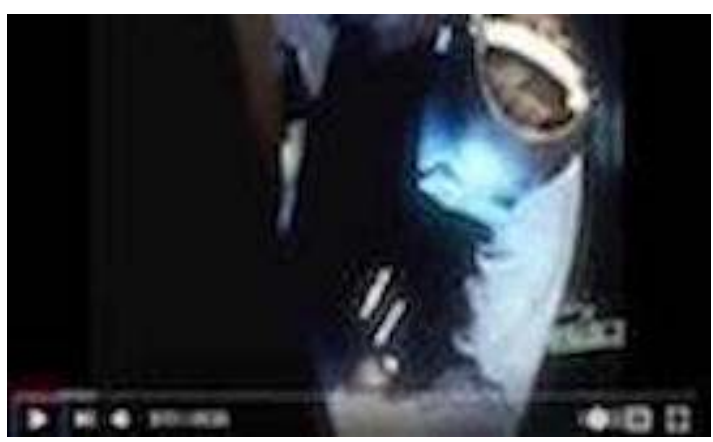

Illustration 42

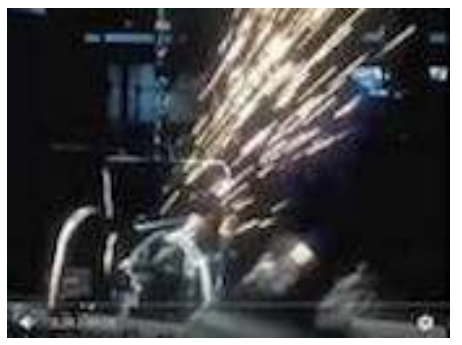

Illustration 43

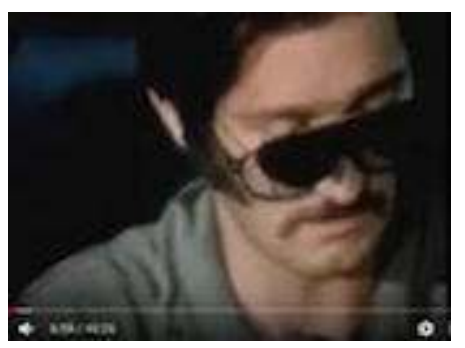


Illustration 44

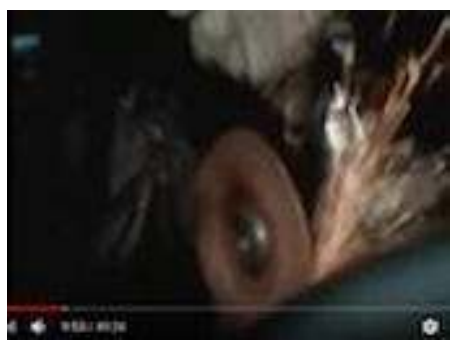

Le parti pris du réalisateur n'est pas de filmer les machines et les merveilles de la technologie. Il est de suivre, au plus près, les corps au travail, les corps en déplacement, les corps aux prises avec les outils et les visages pénétrés, concentrés, absorbés des hommes quand ils sont happés par les dispositifs titanesques de l'usine moderne. Les plans sont rapprochés, saccadés, quasiment morcelés par moment, puis des plans séquences détaillent longuement le bégaiement du geste, le travail qui impose ses séquences obligées, répétées, identiques d'une demi-minute à l'autre, sans fin.

La caméra filme le travail puis, brutalement, elle sort de l'usine. La vie, l'histoire des ouvriers de Peugeot viennent alors faire contrepoint aux images muettes du travail. Via un commentaire en voix off ou à travers la parole de militants, le film s'intéressera à l'habitat des travailleurs, des premiers logements en forme de bidonville aux cités H.L.M. déshumanisantes; il évoquera les pratiques de consommation, s'introduisant dans un des premiers hypermarchés de la région - où un long plan sur les mains de caissières typant sans arrêt le prix des marchandises sur les vieilles caisses enregistreuses rappellera que le taylorisme s'est infiltré dans tous les espaces de travail ; il donnera surtout la parole aux ouvriers, aux ouvrières. Le documentaire se transforme alors en chronique de la tragédie ouvrière. La parole ouvrière fait mal, elle accélère les battements du cœur et arrache des cris de révolte.

Illustration 45

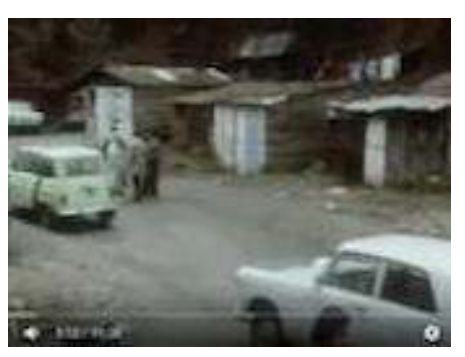

Illustration 46

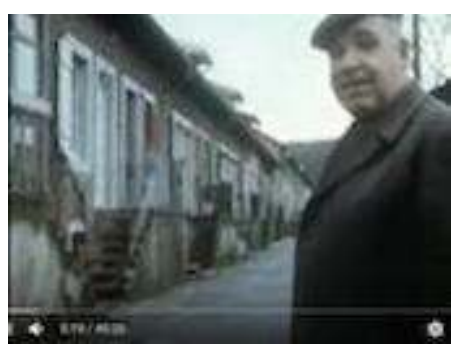




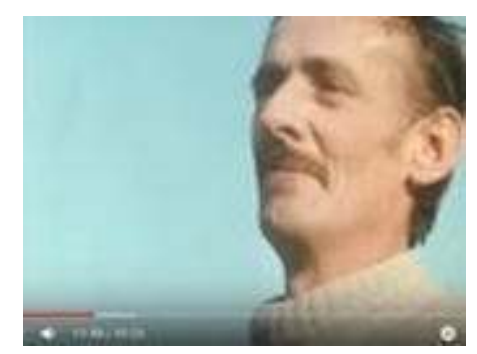

Ce sera d'abord un ouvrier filmé dehors, sous un ciel bleu et froid. Il porte la moustache et un pull blanc à col montant. Visage émacié, il lui manque des dents. Il explique ses horaires de travail, évoque l'horloge qui a fait le «tour du cadran » quand il arrive chez lui, sa journée faite, après une heure de trajet, et les gosses « qu'on n'a pas le temps de voir ", quelle que soit la tournée. Ces gosses, ces cinq gosses, qu'on ne peut pas empêcher de s'amuser quand ils rentrent de l'école, alors qu'il faut se mettre au lit et trouver le sommeil pour pouvoir se lever à 2 h00, quand tout le monde dort. La caméra le prend en pleine face. Le ciel bleu se découpe, dur, derrière lui.

On verra, on entendra, plus tard, une femme - grosses lunettes, chevelure claire, bouclée et volumineuse. Elle est jeune. On la découvre dans sa cuisine, vêtue simplement d'un débardeur. Autour du cou, on lui voit une petite chaîne d'or - elle s'est parée pour l'événement. Elle fume. Elle parle de sa vie, du piège qu'a été sa vie. De la fuite de la prison parentale et de la mise en cage matrimoniale. Elle narre ses désillusions et son monde sans issue. Elle a été gauchiste, " comme tout le monde », et puis ses rêves, ses espérances se sont effondrées. Elle voulait faire du théâtre, sans rien y connaître, et « tout s'est cassé la gueule ». La caméra serre son visage. Ses paroles font mal. L'incommunicabilité marque ses relations sociales, avec ses parents avant, avec son homme à présent. « Christian, ce qu'il veut, il n'arrive même pas à le définir ce qu'il veut ». Et cette phrase comme le couperet d'une guillotine : «Le bonheur, on n'y croit plus de toute façon ». Elle se tait, elle songe, tire sur sa cigarette, s'absorbe dans ses pensées, mordille le dessus de sa main. Vivra-t-elle encore longtemps cette jeune fille pleine d'amertume, mère d'une enfant déjà ? On songe suicide en l'écoutant.

\section{Illustration 48}

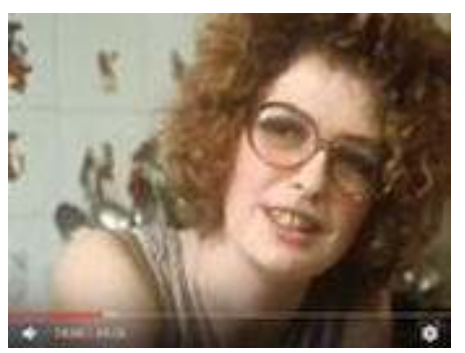


Illustration 49

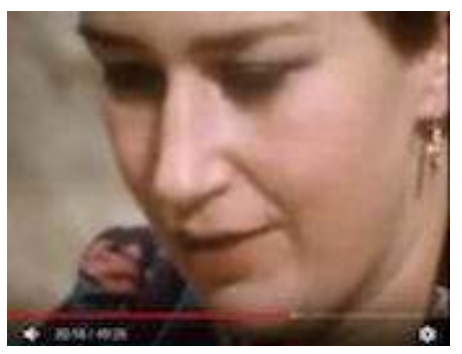

Illustration 50

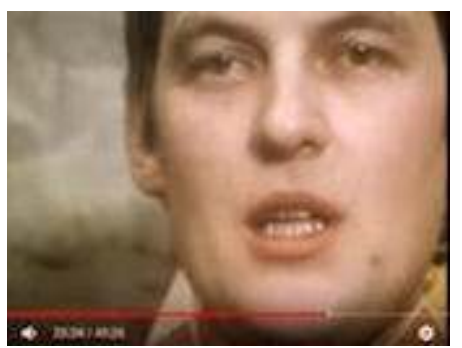

La parole ouvrière semble sortir des tréfonds de l'être. Les questions posées par le réalisateur ne sont jamais montées. $Y$ en a-t-il eu beaucoup ? Etait-il même nécessaire de questionner? Il semble qu'on a ouvert un robinet et que les mots sortent des bouches comme des torrents.

Ainsi, cette autre femme, plus loin. On la voit chez elle. Au calme. Mais, elle, elle n'est pas calme. C'est une syndicaliste de la CGT. Cheveux bruns courts, boucles d'oreille. La caméra cadre son visage. La femme ne regarde personne, se concentre sur son propos. Son élocution est hésitante. Elle se mord les lèvres, bute sur les mots. Elle travaille à la chaîne. Au carrousel de planche de bord 504, en finition, carrosserie. Les postes de femmes enceintes, " soi-disant ». Plus faciles, " soi disant ». "Pas le temps d'aller au WC sans demander au chef ou au contremaître (...) ou bien pour aller se changer, parce qu'il y a des périodes difficiles pour les femmes "... On la reverra cette femme évoquant cette fois les conséquences de cette vie de travail absurde sur les relations de couple. Elle parle des tournées, des horaires impossibles pour faire garder les enfants, obligeant certains ménages à "faire contre-équipe »: un des époux travaille du matin, quand l'autre du soir, se relayant ainsi auprès des enfants, sans jamais se voir. Cela la scandalise, elle s'échauffe, dénonce et s'agace de ne pouvoir mieux s'expliquer - alors qu'elle le fait parfaitement. Sans doute que l'agacement vient de ce qu'elle doit avouer : elle en connaît des couples dans cette situation. « Ils ne s'aiment plus. Ils se supportent. Et pour le gosse, la maison, la pelouse et tout le reste, ils restent ensemble ». Les dégâts du travail débordent la chaîne. Ils contaminent les familles, corrodent les cœurs et les passions. D'autres visages, d'autres mots suivront...

Un des grands moments du film est connu dans les rangs du militantisme - et assurément du monde académique. Il est célébré dans bien des textes où il est question de Sochaux et du travail ouvrier. On y entend une voix off sur des images tournées de nuit aux abords de l'usine, quand arrivent les voitures et les bus pour la tournée du matin. Un homme parle du travail, de son travail, de sa dureté, de ses rythmes et de ses dégâts sur le corps, sur l'esprit, sur les relations de couple. Cet homme, on ne verra pas son visage. C'est Christian Corouge, le film ne le dit pas, mais cela se sait. Corouge est 
célèbre chez les sociologues du travail en raison des travaux réalisés à Sochaux par Michel Pialoux qui est devenu son ami, son confident, son co-auteur aussi, dans certains articles, un livre ${ }^{14}$. Dans ces lointaines années soixante-dix, lui aussi parle de ses désillusions professionnelles, de ses amertumes existentielles, de ce diplôme d'ajusteur qu'il n'a jamais pu faire valoir, du travail qui le mutile, le rend incapable de se servir de ses mains, de nouer les boutons de la petite et de caresser le corps de sa femme. Il évoque la chaîne et la façon dont elle le prive de parole, par la succession de ces journées silencieuses passées les outils à la main, à lutter contre la matière. Et puis il parle de la peur qui est la sienne, la peur que la chaîne l'abaisse encore plus, qu'après les mains, elle s'attaque au reste du corps, le plonge définitivement dans le silence, le mutisme, une manière de folie qu'il redoute et qu'il voit poindre dans le fait qu'il n'arrive plus à lire, qu'il n'en a plus envie. La chaîne l'a privé de son humanité. Il le sait. Il a peur. L'image se fait plus claire, le jour se lève, et l'usine, toujours, absorbe sa chair humaine. Elle arrive en bus, en voiture, à deux-roues, à pied aussi et l'usine avale tout. "C'est de la survie qu'on fait ». Mais Corouge veut lutter encore et ne pas « se laisser bouffer » par Peugeot. Depuis l'âge de 18 ans, il lutte. Combien de temps encore?

Illustration 51

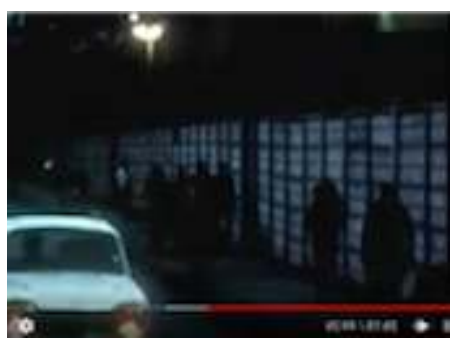

Illustration 52

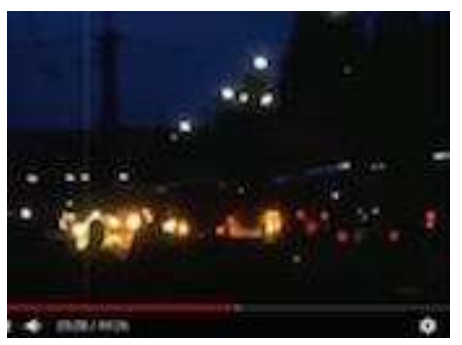

Illustration 53

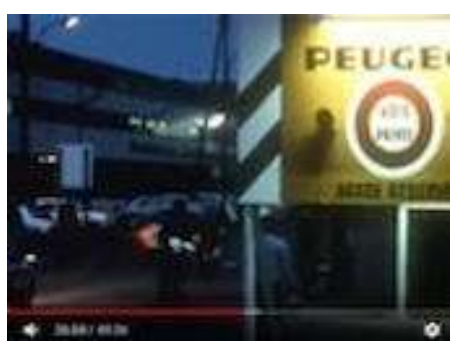

Muel, évidemment, ne pouvait faire l'impasse sur la question des luttes ouvrières. La caméra quitte alors le temps présent, les ateliers et le récit des souffrances pour tenter de ressusciter le souvenir des combats ouvriers. 

réalisation du film Sochaux, 11 juin 1968 ( $c f$. Muel, 2000). La bande son laisse entendre un bruit de manifestation, des détonations, tandis qu'on voit des scènes d'occupation. La caméra filme aussi des graffiti sur les murs : un ouvrier avec la peau d'un lion sur le dos ; un ouvrier torse nu, musclé, armé d'un trident, nouveau gladiateur, qui repousse un lion tombant à la renverse. Tous les symboles de la lutte sont là, avec les images, les photos et la bande son qui crache les décibels d'une manifestation avant de s'adoucir pour laisser la place à un commentaire off, neutre, objectif.

La grève, il faut en parler aussi, car les images ne montrent pas tout, ne disent pas tout. En 68, les ouvriers tenaient l'usine, mais le 11 juin, c'est l'assaut des forces de l'ordre. Le commentaire nous apprend qu'on relèvera deux morts parmi les manifestants, Henri Blanchet, 49 ans, Pierre Bellot, 24 ans tués par balle ou à la suite de l'explosion d'une grenade offensive (ce n'est arrivé nulle part ailleurs des morts de ce genre) ${ }^{15}$.

Plusieurs ouvriers ont eu un pied, une main arrachés par les explosifs. On a compté 150 blessés. Images d'ouvriers sur l'écran, images de CRS en escadron, fusil à l'épaule, de CRS chargeant, courant, tirant. On pense au Chili, à l'Amérique du sud. Mais c'est la France.

Après ces images terribles, et un détour par de nouveaux témoignages consacrés aux stratégies patronales mises en place pour désamorcer la puissance salariale (recours massif aux immigrés, leur placement judicieux le long des chaînes aux côtés des Français afin de casser les ententes traditionnelles et d'empêcher l'échange; l'obligation d'adhésion à la $\mathrm{CFT}^{16}$, le syndicat maison, etc.) ou aux exactions des nervis de ce syndicat contre des colleurs d'affiches CGT (vision terrifiante de ce colosse qu'est René Ledigherer, ouvrier-militant- medvedkinien, sur son lit d'hôpital avec ses dizaines de points de suture), le documentaire s'achemine vers sa conclusion

\section{Illustration 54}

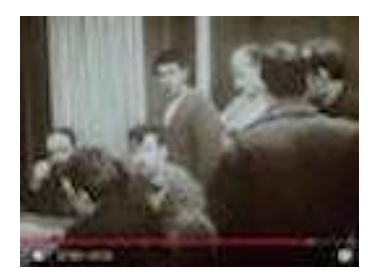

Illustration 55

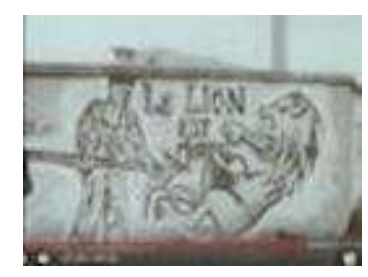


Illustration 56

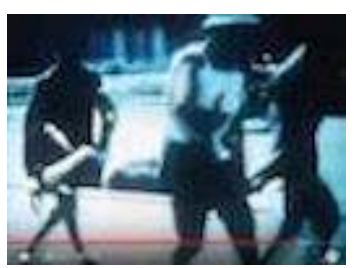

Illustration 57

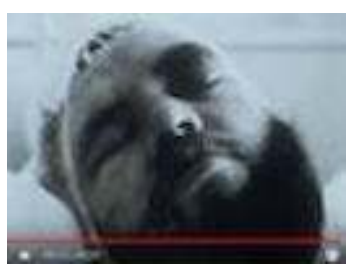

On découvre les ouvriers, au cœur de l'été, partager un moment de convivialité militante au bord d'un étang. Nous sommes à une fête politique, dans un camping improvisé. C'est l'expression de la légendaire camaraderie ouvrière qu'on montre, le peuple resserré sur lui- même au milieu des rires, de la nourriture, de l'alcool et de l'enthousiasme. Ledigherer, plus gargantuesque que jamais, grille de la viande sur un barbecue. Une chanson s'élève dans l'air. La caméra cadre un groupe d'ouvriers reprenant en cœur les paroles d'une chanson interprétée par un jeune et sa guitare.

«Les cadences infernales... À la boîte !... La production, la production...

Solidaritééééé ». Les visages sont sérieux. Les ouvriers fument, leurs femmes

fument. Tous chantent : « Non, nous ne rêvons pas, nous referons un monde ».

51 Mais les dernières images sont celles de l'usine, du bruit et des visages tendus des ouvriers à leur poste de travail dans le vacarme des presses de 200 tonnes qui martèlent l'acier. Ces images sont terribles. Ce ne sont pas les tôles que les presses écrasent, ce sont les hommes qui se tiennent à leurs côtés, silencieux et mécaniques. Organes passifs enchaînés aux presses. Ce film est, peut-être, la chronique d'une défaite, l'épitaphe d'un monde englouti par les années 1980 et le néolibéralisme qui va se répandre et dont l'auteur du film, à l'écoute des ouvriers, a peut-être pressenti l'arrivée. C'est assurément ce qu'on peut inférer de ces mots de l'auteur : «Avec le sang des autres : plus encore qu'un film sur les conditions de travail, c'est un film sur la violence. C'est comme une leçon d'histoire, très sombre, où on voit les années passer et rien ne s'arranger pour la classe ouvrière, au contraire $»^{17}$. Qu'il ait conclu en disant : «Ça peut donner envie de se battre ", ne change rien au regard désenchanté porté sur l'évolution socio-économique et la condition ouvrière.

\section{Conclusion}

Il n'est pas nécessaire d'insister beaucoup sur les divergences qui marquent ces deux documents. C'est vrai de l'intention portée par les deux films comme des choix d'écriture cinématographique. Dans un cas, on met au service de la cause patronale d'édification des populations les ressources d'un protocole cinématographique parfaitement maîtrisé, empruntant aux méthodes d'écriture, de filmage et de montage les plus académiques. Du point de vue des objectifs qui sont les siens, ce film est une 
réussite. La prouesse centrale de ce documentaire est d'avoir réussi, par le choix des commentaires, à quasiment occulter la présence humaine dans les ateliers, tout en montrant à chaque image un homme ou une femme au travail. Cette occultation du travailleur va de pair avec la dénégation de toute dimension socio-politique au travail. L'homme, la femme ne sont qu'un des passages «techniques » nécessaires mais subalternes du démiurgisme entrepreneurial, de la soif créative des capitaines d'industrie, des prouesses techniques des grandes sociétés industrielles.

Dans l'autre document, à l'inverse, le travail, les hommes sont au centre de l'attention. Ce que le film met au centre du débat, c'est l'exploitation du travailleur dans le cadre de la compétition capitaliste et la mise à sac de son existence dans et hors le travail. Le travailleur est filmé avec une patience, une attention clinique, quasi entomologique, grâce à des images longues, précises, mais le spectateur le suit aussi en dehors des ateliers, où la caméra, le micro lui redonnent un visage singulier et une parole. Les ouvriers ne sont plus des éléments mutiques du décor industriel. On les filme chez eux et à hauteur d'homme. On découvre leur habitat, leur lieu de consommation, l'histoire collective qui est la leur. Les commentaires off donnent du sens aux images, se font historiques, sociologiques, critiques. On demande surtout aux ouvriers eux-mêmes de parler de leur vie et ils le font, déposant dans nos âmes le poids mort de leurs rêves avortés, de leurs aspirations déçues et de leur santé saccagée. Le travailleur devient un être doué d'une voix et, stupéfaction, il pense, il souffre aussi. La caméra ne se préoccupe pas de rater ses plans, ses cadrages, ses zooms et sa mise au point - du reste, les images prises dans l'usine sont d'une justesse documentaire et d'une beauté plastique incontestables. L'essentiel est ailleurs, dans l'intensité dramatique de l'affrontement de classe tel qu'il se présentait à l'époque et que les hommes et les femmes qu'on filme au travail, qu'on suit dehors pour entendre leur parole, nous aident à comprendre. Le diagnostic de l'auteur, quoiqu'il n'ait pas négligé d'insister sur les luttes menées par les ouvriers pour tenter de changer le travail, de changer la vie, ce diagnostic s'avère sombre. On ressort du visionnage écrasé. Sans voix. Et les trente années écoulées depuis le tournage ne semblent pas devoir remettre en cause les conclusions de l'auteur.

Ces deux documents méritaient d'être traités de façon comparative : ce bi-focalisme permet de redonner de la profondeur à la vie ouvrière, de la lester d'une charge émotionnelle juste que le premier document entendait réserver aux affects heureux d'une humanité débordante d'énergie créatrice. Au moment de la réalisation du film de Muel, l'usine Peugeot voisinait l'acmé de sa puissance humaine. Plus de quarante mille salariés se rendaient chaque jour sur le site pour assembler des automobiles, 200000 personnes travaillaient directement ou indirectement pour le groupe Peugeot dans la région (Hatzfeld, 2002). À Sochaux, aujourd'hui, on ne parle plus que de dix mille salariés, dont un peu plus de la moitié d'ouvriers, quelques deux mille employés, techniciens et agents de maîtrise... Ils produisent environ 1500 véhicules/jour, soient 380000 véhicules par an, et des modèles dont la complexité et la variété ont explosé. Le vingt millionième véhicule de la marque est sorti de Sochaux en 2010 ( $c f$. Lamard et Belot, 2007). Il serait évidemment intéressant de comparer le document de Muel avec un travail du même type, plus récent, afin de mesurer ce qui a changé et, sous ce changement, ce qui est demeuré immuable : la domination et l'exploitation du travail ${ }^{18}$. 


\section{BIBLIOGRAPHIE}

Corouge Ch., Pialoux M. (1985), « Chroniques Peugeot », Actes de la Recherche en Sciences Sociales, vol. $60, \mathrm{n}^{\circ} 1, \mathrm{p} .72-74$.

Hatzfeld N. (2002), Les gens d'usine. 50 ans d'histoire à Peugeot-Sochaux, Paris, Editions de l'Atelier/ Éditions ouvrières.

Joubet J.-L., Hatzfeld N. (2002), « Poissy : de la CGT à la CFT. Histoire d'une usine atypique », Vingtième siècle. Revue d'histoire, vol. 1, n 73, p. 67-81.

Hatzfeld N., Rot G. et Michel A. (2004), « Le travail en représentation dans les films militants. Caméras et micros dans les usines automobiles, 1968-1974 », Histoire et sociétés, n 9, 1er trimestre, p. 118-131.

Hatzfeld N., Rot G. et Michel A. (2006), «Filmer le travail au nom de l'entreprise ? Les films Renault sur les chaînes de production ", Entreprises et Histoire, n 44, septembre, p. 25-42.

Hatzfeld N., Rot G. et Michel A., (2007a), « Quand la chaîne fait son cinéma. Regards sociologiques et historiques sur une représentation cinématographique du travail (chantier) », Terrains \& travaux, vol. 2, n 13, p. 189-202. [En ligne]. URL : https://www.cairn.info/revue-terrains-ettravaux-2007-2-page-189.htm

Hatzfeld N., Rot G. et Michel A. (2007b), « Le travailleur de l'automobile au cinéma. Montageremontage d'un personnage pluriel », Revue d'histoire des chemins de fer [En ligne], mis en ligne le 10 mai 2011, consulté le 29 janvier 2018. URL : http://journals.openedition.org/rhcf/141

Hatzfeld N., Rot G. et Michel A. (2009), « L'ouvrier en personne, une irruption dans le documentaire », Le mouvement social, Janv.-mars, La Découverte, p. 67-79.

Joubet J.-L., Hatzfeld N. (2004), « Les conflits Talbot, du printemps syndical au tournant de la rigueur, 1982-1984 ", Vingtième Siècle. Revue d'histoire, vol. 4, n 84, p. 151-160.

Lamard P., Belot R. (2007), Peugeot à Sochaux, des hommes, une usine, un territoire, Panazol, Editions Lavauzelle.

Linhart R.,( 1978), L'Etabli, Paris,Éditions de Minuit.

Muel B. (2000), « Les riches heures du groupe Medvedkine (Besançon-Sochaux, 1967-1974) », Images documentaires, $\mathrm{n}^{\circ}$ 37-38, 1er et 2e trim., p. 10-35.

Pialoux M., Corouge Ch. (2011), Résister à la chaine. Dialogue entre un ouvrier de Peugeot et un sociologue, Marseille, Éditions Agone.

Service Inventaire et Patrimoine, Région Franche-Comté (2014), Le Pays de Montbéliard et son patrimoine industriel, Lyon, Lieux-dits.

Vigna X. (2006), « Les Groupes Medvedkine, Besançon et Sochaux », Cahiers d'histoire, Revue d'histoire critique, $\mathrm{n}^{\circ}$ 99. [En ligne], mis en ligne le 22 juin 2009, consulté le 24 janvier 2018. URL : http://journals.openedition.org/chrhc/743

\section{NOTES}

1. Cf. https://www.youtube.com/watch?v=atP0kFM-Xko Production: Les Films Pierre Boyer, Paris. Images : René Gosset ; caméra : Didier Tarot, assisté de Christiane Broïdo et Daniel Jannin ; montage : Maurice Bonin, sur une musique originale de Roger Roger (sic) ; 58 mn. 
2. C'est la date du moins qu'annonce la personne qui a posté ce document, toutefois dans les commentaires plusieurs personnes contestent cette donnée, indiquant par des remarques convaincantes que le document est plus vraisemblablement de 1957. Cette date est indirectement corroborée par le commentaire accompagnant le film qui parle de l'usine de Beaulieu comme ayant été érigée « il y a un siècle », en 1857. J'incline à penser, sans en avoir de preuve formelle, que ce film est donc au moins de cette année-là.

3. Il existe très peu de traces de ce réalisateur sur le net (http://data.bnf.fr/11454849/ pierre_boyer/http://www.linternaute.com/cinema/pierre-boyer/ et http://www.imdb.com/ company/co0311253/). Boyer (1906-1964) est présenté sur le site de la BNF comme «Critique, producteur et réalisateur de cinéma - Rédacteur en chef de la revue Ciné-amateur ». Il semble avoir signé avec Pierre Faveau plusieurs opuscules sur les techniques cinématographiques ou du matériel de tournage, ainsi que des textes sur l'Algérie. De lui, on trouve trace d'un film [documentaire ?] de 32', titré Mirage, de 1937. Deux documentaires d'Ed. Molinaro, de 1953, sont réalisés par la société « Les films Pierre Boyer » (ibid.). Rien sur ce film chez Peugeot.

4. Le travail de Boyer doit sans doute beaucoup à un film de 40' tourné aux Usines Citroën du quai de Javel, par Léon Poirier, disponible sur le net (sans date), intitulé Autopolis. La cité de l'automobile: https://www.youtube.com/watch?v=DhyvYN42j3o. Consacré à la fabrication des «Tractions avants» (commencée en 1934, stoppée en 1957, ce qui me fait dire que ce film est antérieur à celui consacré à la 403), on y retrouve exactement les mêmes recettes : apologie de la machine, mise en valeur du génie organisateur de la marque, plans aériens, longs plans fixes sur les outils au travail, travellings, plongées, contre-plongées, etc., musiques entraînantes de toutes natures et même oubli de l'homme au travail, même mépris pour la question des conditions de travail.

5. Une des questions qui vient immédiatement à l'esprit pendant le visionnage du film est : comment a-t-il pu tourner ces images au cœur même de l'usine, comment a-t-il obtenu l'autorisation de faire ces prises de vue quand on sait le traitement qu'il leur a réservé ? Dans un texte évoquant son itinéraire de cinéaste en Franche-Comté, on trouve écrit cette indication sibylline : « Nous avons réussi, par un tour de passe-passe dont nous étions assez fiers, à obtenir les images des chaînes Peugeot, celles que nous voulions, les lieux précis et les angles de prise de vues que ceux qui y travaillent nous avaient décrits. » (cf. Muel, 2000, p. 33). Ces mots soulignent bien qu'il y avait un problème à tourner ces images, mais ne disent pas comment il a été contourné. Une discussion récente avec Henri Traforetti, du groupe Medvedkine- Besançon, révèle que Muel aurait profité de la présence d'une équipe de tournage de la $\mathrm{BBC}$, prétextant réaliser un film sur les progrès techniques dans l'industrie européenne, pour faire réaliser les images voulues dans les ateliers. Muel n'est donc pas derrière la caméra quand elle est dans l'usine (information reprise par Hatzfeld et alii, 2007a, $c f$. leur note 11).

6. La société ISKRA (Image, Son, Kinescope et Réalisations Audiovisuelles, étincelle en russe - c'est le nom du journal lancé par les Bolcheviks au moment de la révolution russe), anciennement SLON (Service de Lancement des Euvres Nouvelles, Eléphant, en russe), productrice des films des groupes Medvedkine dispose de son site, où sont présentés les 160 films de son catalogue : http:// www.iskra.fr/fron ; elle y a ajouté en 2015 un documentaire sur les abattoirs intitulé «Saigneurs » (92'), dont la version courte de 52', s'intitule « Avec le sang des hommes ». Impossible de ne pas penser au film de Muel. Pour le titre de son film, celui-ci s'est peut-être inspiré du documentaire de Georges Franju, de 1948, sur le travail dans les abattoirs : Le sang des bêtes...

7. Document accessible sur le net : https://www.imagesdocumentaires.fr/IMG/pdf/Idoc37_38.pdf Le VD74DDSQ\&cad=rja Ce numéro d'Images documentaires constitue un vivier d'informations pour ce qui touche aux groupes Medvedkine, donnant la parole à plusieurs témoins de l'époque, en plus de Muel (Henri Traforetti, Georges Binetruy, Michel Pialoux), et contient par ailleurs une liste de 200 films sur le monde ouvrier. Le site «Les trains de lumière» offre un historique et la 
filmographie complète et commentée des Groupes Medvekine: https://sites.google.com/site/ lestrainsdelumiere/groupes-medvekine

8. Il aurait existé à la même époque plus d'une vingtaine de collectifs de cinéma militant en France, le groupe Medvedkine y occupe une place singulière (cf. Vigna, 2006).

9. La technique de portage, quoique mobilisée parfois, ne pouvait guère être envisagée avant les années 1970 en raison de la taille et du poids des matériels de filmage. En France, Jean-Pierre Bauviala développe, à partir de 1973, les premiers modèles de caméra permettant le portage à l'épaule (caméra Aäton 7A). Ces questions relatives aux matériels d'enregistrement des images et des sons sont centrales dans l'émergence du film documentaire - a fortiori militant (Hatzfeld et alii, 2009).

10. Étrangement, l'usine d'Hérimoncourt, la plus ancienne de la famille (cf. Service Inventaire et Patrimoine, 2014), n'est pas présentée.

11. Sochaux ouvre en 1912. Le document annonce les chiffres suivants pour l'époque du tournage : 17000 salariés, 245 hectares d'emprise au sol, 150 trains de charbon et $200000 \mathrm{Kw} / \mathrm{h}$ consommés. L'énergie est produite sur place et commandée à partir d'une salle « entièrement automatisée » dont le documentaire offre quelques vues intérieures.

12. Ce procédé d'occultation de l'homme au profit de la machine semble constant dans les films d'entreprise, $c f$. Hatzfeld et alii, 2007a.

13. https://www.youtube.com/watch?v=L8GYGToGWJY

14. C'est d'ailleurs grâce à $B$. Muel que les deux hommes se sont rencontrés. Pour un aperçu de leur travail, $c f$. en particulier leur dernière collaboration : Pialoux, Corouge, 2011. Dans ce livre, on apprend qu'à un moment éprouvant de sa vie, Ch. Corouge a tenté de mettre fin à ses jours ; ou Corouge et Pialoux, 1985 (entre autres). On peut aussi se référer à un entretien disponible sur le net avec Tangui Perron, cette fois, où Corouge évoque entre autres choses sa contribution à Avec le sang des autres et l'accueil qui lui a été réservé, en particulier du côté du PC (cf. http:// www.article11.info/?Christian-Corouge-Quand-on-est-en).

15. http://www.liberation.fr/grand-angle/2008/05/30/juin-meurtrier-a-sochaux_72909

16. Confédération Française du Travail. Issu de la Confédération Générale des Syndicats Indépendants, elle est fondée en 1959 et devient la Confédération des Syndicats Libres, en 1977, après qu'un militant de la CGT a été abattu par un de ses membres dans une fusillade. Elle se saborde en 2002. Très présent dans l'automobile, Robert Linhart écrit à son propos, en 1978 : « Nous avons parmi nous des mouchards de toutes nationalités, et surtout le syndicat maison, la C.F.T., ramassis de briseurs de grèves et de truqueurs d'élections. Ce syndicat jaune est l'enfant chéri de la direction : y adhérer facilite la promotion des cadres et, souvent, l'agent de secteur contraint des immigrés à prendre leur carte, en les menaçant de licenciement, ou d'être expulsés des foyers Citroën. » (cf. Linhart, 1978, p. 67). Pour un aperçu de l'histoire et de l'évolution de la CFT, $c f$. Joubet et Hatzfeld, 2002/1, p. 67-81, où l'on assiste à l'ascension de la CFT chez Simca, avant son essaimage dans l'automobile. Dans un autre article, les deux auteurs précisent: "Supplantant les syndicats autonomes de l'immédiat après-guerre, la CFT - devenue CSL constituait une tradition de la droite sociale française (...) Elle s'était implantée chez Peugeot après 1968, sans devenir dominante, et était apparue fortuitement dans certaines usines Renault. (...) Ce syndicat corporatiste accueillait tous les types de salariés, de l'os à l'ingénieur. Dirigé par des hommes liés aux partis de droite et d'extrême droite, auxquels il fournissait des colleurs d'affiche, voire, parfois, des candidats aux élections locales, il prétendait à l'exclusivité et combattait avec énergie la CGT. » (cf. Loubet et Hatzfeld, 2004/4, p. 151-160).

17. https://www.disparaissezlesouvriers.fr/le-sang-des-autres

18. Ce travail pourrait être le documentaire de 53' de Laurence Jourdan : Sochaux, cadences en chaînes, daté de 2010 (https://www.youtube.com/watch?v=Ak0XAJPKRys). On peut y déceler les permanences et les changements qui ont marqué l'organisation du travail et les logiques de production. Des images de B. Muel et du groupe Medvedkine y sont utilisées pour des rappels 
historiques (peut-être aussi un tout petit extrait de " En passant par la Franche-Comté ») et on y retrouve évidemment un témoignage de Christian Corouge.

\section{RÉSUMÉS}

Le texte vise l'analyse comparative de deux documentaires disponibles sur le net consacrés aux usines Peugeot de Sochaux. D'un côté En passant par la Franche-Comté, de Michel Boyer (1954 [1957 ?], 54') ; de l'autre, Avec le sang des autres, de Bruno Muel (1974, 50'). Tout distingue ces deux films: la date de réalisation, les intentions idéologiques, les moyens techniques et financiers mis en œuvre. A travers leur décryptage minutieux, nous tenterons de mettre en lumière les choix scénaristiques et d'écriture cinématographique retenus par les auteurs pour servir leur projet. Apologie du génie industriel d'une famille dans la négation du travail humain contre témoignage socio-historique des dégâts liés à la mise au travail de la ressource humaine de toute une région, tels semblent être les termes de l'opposition. Nous nous attacherons à montrer les artifices cinématographiques privilégiés par chaque auteur.

This text sets out a comparative analysis of two documentaries - available on the internet focused on Peugeot's factories in Sochaux. The first one is titled «En passant par la FrancheComté » (Throuh Franche-Comté), directed by Michel Boyer (1954 [1957 ?], 54'); the second one is titled «Avec le sang des autres" (With the blood of the others), directed by Bruno Muel (1974, 50 '). Everything is different about these movies : the date of production, the ideological purposes, the financial and technical means implemented. By deciphering both documentaries meticulously, we will try to highlight the decisions taken by the directors regarding the storylines and the writing in the best interest of their project. Apology of the industrial genius of a family in the negation of human labor against socio-historical testimony of the damage related to the putting into work of the human resource of a whole region, that is the opposition. We will focus on disclosing the special cinematic devices og each director.

\section{INDEX}

Mots-clés : travail, Peugeot, Sochaux, Medvedkine, cinéma documentaire, engagement

Keywords : work, Sochaux, Peugeot, Medvedkine, commitment, documentary

\section{AUTEUR}

\section{SALVATORE MAUGERI}

Salvatore Maugeri est docteur en sociologie, diplômé de l'université Paris X-Nanterre, maître de conférences à l'Université d'Orléans. Il consacre sa recherche au travail et à sa gestion. Il est membre-fondateur du RT 30/Sociologie de la gestion de l'Association Française de Sociologie, et fait partie du comité de rédaction de la Nouvelle Revue du Travail, une revue pluridisciplinaire en ligne consacrée aux problématiques du travail et de l'emploi. En marge de ses articles de recherche, il a publié plusieurs ouvrages de vulgarisation et coordonné quelques recueils de 
textes scientifiques touchant à ses objets scientifiques. Il a traduit de l'italien et préfacé un ouvrage de G. Kaczinski, consacré à Florian Znaniecki, sociologue polono-américain à la fois célèbre et méconnu (La connaissance comme profession, L'Harmattan, 2008). 